\title{
The enterohepatic circulation of bile acids as they relate to lipid disorders
}

\author{
R. HERMON DOWLING
}

From the MRC Intestinal Malabsorption Group, Department of Medicine, Royal Postgraduate Medical School, Ducane Road, London W12

Bile acids ${ }^{1}$ play a fundamental role in lipid metabolism. They solubilize the products of lipolysis thereby facilitating digestion of triglycerides and the absorption of monoglycerides and fatty acids, and their presence is mandatory for the absorption of cholesterol and the fat-soluble vitamins. The enterohepatic circulation of bile acids ensures that adequate concentrations of these biological detergents are available when needed in the intestinal lumen.

This paper, therefore, begins by reviewing the normal enterohepatic circulation of bile acids as illustrated by studies in the rhesus monkey. The influence of diet and particularly of dietary fat on this circulation is then discussed with emphasis on some further recent studies in the monkey which suggest a possible mechanism for the hypocholesterolaemic action of polyunsaturated fats. Finally, some effects of interrupting the bile acid enterohepatic circulation are considered. These include the development of hyperoxaluria and occasionally renal oxalate stone formation, and the formation of a bile supersaturated with cholesterol and the subsequent development of cholesterol gallstones.

\section{The Enterohepatic Circulation of Bile Acids}

The normal bile acid enterohepatic circulation is illustrated diagrammatically in figure 1.

The primary bile acids, cholic acid and chenodeoxycholic acid (fig 2) are synthesized in the liver from cholesterol and after conjugation with the amino acids glycine and taurine are temporarily stored and concentrated in the gallbladder. Then, in response to a fatty meal, the bile acids pass into the upper intestine where they play their important role in promoting the digestion and absorption of fat. Reabsorption of bile acids occurs mainly in the ileum by an active transport mechanism, and they return to the liver via the portal vein thus completing the enterohepatic circulation. The intestinal absorption

\footnotetext{
'The terms 'bile acids' and 'bile salts' are used loosely and interchangeably. The extent to which a bile acid is present as an undissociated acid or as an ionized salt depends on the pKa of the individual bile acid and the environmental $\mathrm{pH}$.
}

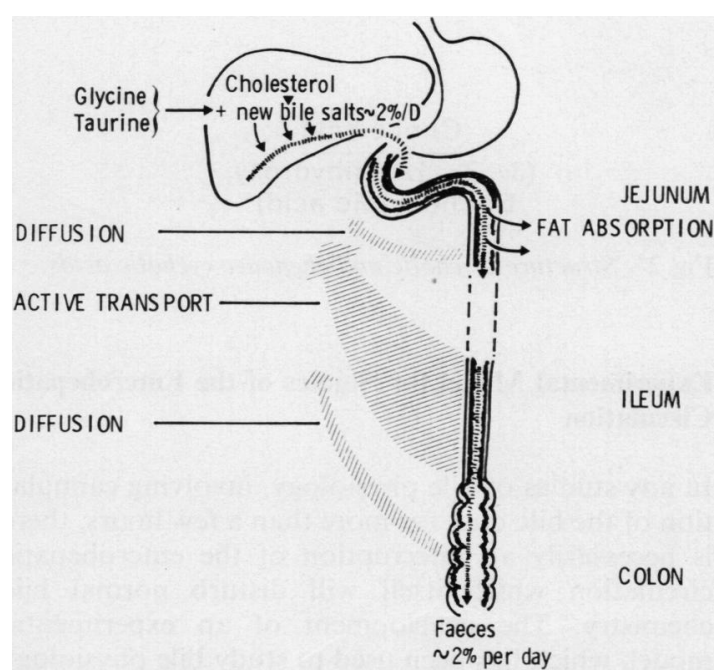

Fig 1 The normal bile acid enterohepatic circulation.

of bile acids is extremely efficient, about 95 to $98 \%$ of the total circulating bile acids being absorbed each day. Only a small quantity escapes reabsorption and is excreted in the faeces so that, to maintain the status quo, the liver has only to synthesize enough bile acids to match the faecal loss.

The primary bile acids, cholic acid and chenodeoxycholic acid (fig 2), are first deconjugated and then further metabolized by intestinal bacteria which remove an hydroxyl group from the 7-position of the steroid molecule, thus forming deoxycholic acid from cholic acid, and lithocholic acid from chenodeoxycholic acid. Lithocholic acid is highly insoluble and is largely excreted in the stools, presumably in a crystalline form adherent to dietary fibre. In contrast, deoxycholic acid is partially reabsorbed from the colon and passes to the liver where it is conjugated with glycine or taurine before joining the primary bile acids already present in the enterohepatic circuit. There are thus six major bile acids in human bile-the glycine and taurine conjugates of cholic, chenodeoxycholic, and deoxycholic acids. 
<smiles>CC(CCC(=O)O)C1CCC2C3C(O)CC4C[C@@H](O)CCC4(C)C3CC(O)C12C</smiles>

Cholic acid

$(3 a, 7 a, 12 a-$ trihydroxy, $5 \beta$-cholanoic acid)

Fig 2 Structure of cholic and chenodeoxycholic acids.

\section{Experimental Model for Studies of the Enterohepatic Circulation}

In any studies of bile physiology, involving cannulation of the bile duct for more than a few hours, there is necessarily an interruption of the enterohepatic circulation which itself will disturb normal bile chemistry. The development of an experimental model, which has been used to study bile physiology in the rhesus monkey (Dowling, Mack, Picott, Berger, and Small, 1968; Campbell, Burgess, Roberts, and Dowling, 1972), has largely overcome this problem. The experimental design is illustrated diagrammatically in figure 3 . In animals trained to sit in a restraining chair before surgery, the common bile duct was ligated distally and a T-tube inserted in the common duct, where possible below the entry of the cystic duct, so that the cannula drained both hepatic and gallbladder bile. The drops of bile then passed between the light source and photoelectric cell of an electronic stream splitter, which could be preset to divert, by means of movable funnels, every third, fifth, tenth, or twentieth drop of bile into a sampling tube. The remainder of the bile passed into a small cup balanced in such a way that only a few drops of bile were required to close a switch, activate a pump, and return the bile to the upper intestinal tract. In each animal $5 \%$ of the bile was collected until steady state conditions were reached some two to four weeks after surgery. Since $95 \%$ of bile was returned to the upper intestine, a virtually intact enterohepatic circulation was maintained, but a representative sample of bile was available for analysis together with an accurate measure of the

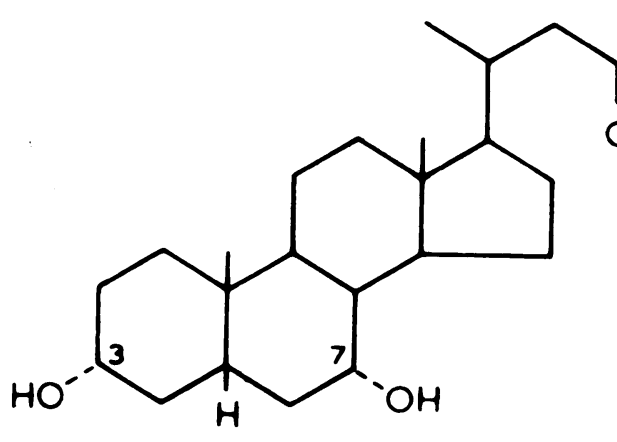

\section{Chenodeoxycholic acid (3a,7a-dihydroxy, $5 \beta$-cholanoic acid)}

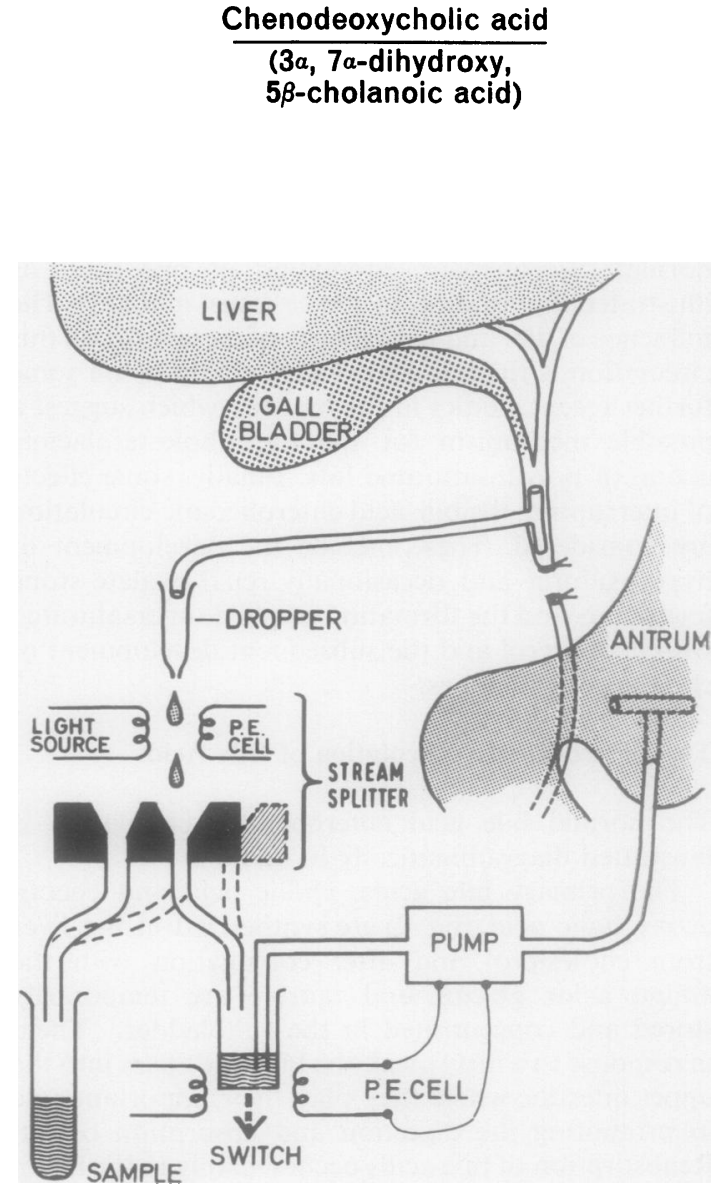

Fig 3 Experimental model for controlled interruption of the enterohepatic circulation in the rhesus monkey. Bile from a chronic bile fistula is returned to the antral region of the stomach through an electronic stream splitter which diverts variable percentages of the biliary output to a sampling tube (Dowling et al, 1968). 
volume of bile produced during any given period of time. Bile composition was then studied in the steady state at $10,20,33,66$, and $100 \%$ interruption of the enterohepatic circulation.

Although originally designed to examine the effects of chronic interruption of the enterohepatic circulation, the use of this model enables one to define all the various components of the normal enterohepatic cycle: (1) bile acid secretion rate; (2) bile acid synthesis; (3) the mechanism of the feedback or autoregulation of bile acid synthesis; (4) the efficiency of bile acid absorption from the intestine; $(5)$ the bile acid pool size; and (6) the number of enterohepatic cycles per day.

BILE ACID SECRETION

Bile acid secretion is defined as the total amount of bile acids passing through the bile duct during a 24-hour period. This consists mainly of reabsorbed bile acids, some $2-5 \%$ of the total daily output being produced by hepatic synthesis de novo.

\section{BILE ACID SYNTHESIS}

In the steady state, bile acid synthesis equals loss. With the experimental model described above, daily measurements of bile acid secretion obtained over at least a seven-day period established that the animals were indeed in a steady state. In this situation bile acid loss is the sum of that excreted in the faeces plus that removed with the stream splitter. By measuring faecal bile acid excretion, therefore, synthesis may be calculated.

CONTROL OF BILE ACID SYNTHESIS

Since the total daily bile acid output is known and faecal excretion may be measured, the amount of bile acids which are reabsorbed and returned to the liver is obtained by simple subtraction. The results of bile acid synthesis plotted against the amount returning to the liver are shown in figure 4 . By extrapolation, if as many as 10.3 millimoles of bile acids per 24 hours were to return to the liver, synthesis would be completely inhibited; with increasing degrees of biliary diversion the amount of bile acids returning to the liver falls, and there is a linear increase in synthesis until less than 7 millimoles of bile acids return to the liver each 24 hours, when synthesis is working maximally. It seems, therefore, that the stimulation of bile acid synthesis is sensitively controlled over a narrow range of bile acid return to the liver.

\section{BILE ACID POOL SIZE}

Two principal methods of measuring the bile acid pool are currently in use-an isotope dilution technique which is used in man, and a more direct

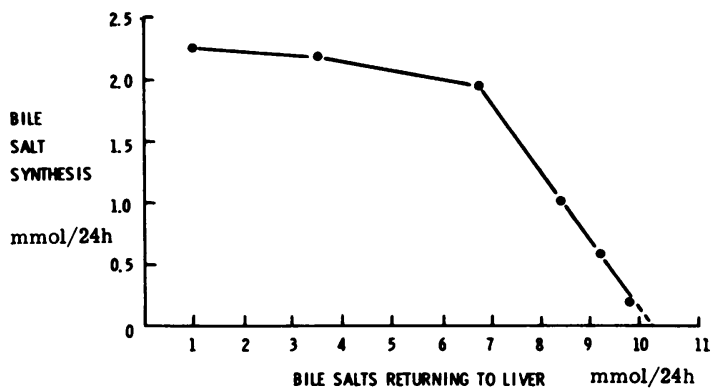

Fig 4 Feedback control of bile acid synthesis: bile acids returning to the liver plotted against bile acid synthesis.

'washout' technique based on bile fistula studies which is suitable for experimental animals.

\section{The isotope dilution technique (Lindstedt, 1957)}

This involves administration of bile acid radioisotopes either orally or intravenously, which are assumed to be uniformly distributed in the endogenous circulating bile acid pool. Duodenal intubation is then performed daily for a four- or five-day period, and the decline in bile acid specific activity plotted against time. On a semi-logarithmic plot a straight line is obtained, indicating first-order kinetics, and by extrapolation of this line to zero time, the initial specific activity is obtained. Knowing the amount of radioactivity administered to the patient, the bile acid pool size may be calculated.

\section{The washout technique (Eriksson, 1957, as modified} by Myant and Eder, 1961)

The principles of this technique are illustrated diagrammatically in figure 5. Immediately after the insertion of a cannula in the common bile duct, the bile acid secretion rate is initially maintained by bile acids which were present in the enterohepatic circulation at the time of fistulation. The secretion rate then falls rapidly to reach a low point, which is thought to represent the basal rate of hepatic bile acid synthesis. The amount of bile acids secreted from the time of insertion of the cannula until the low point is reached provides a direct measure of the bile acid pool.

\section{NUMBER OF ENTEROHEPATIC CYCLES PER DAY}

Knowing the previous steady state bile acid secretion rate (in the rhesus monkey model), the number of enterohepatic cycles may be calculated by dividing the secretion rate by the pool size.

An example of how these various components of 


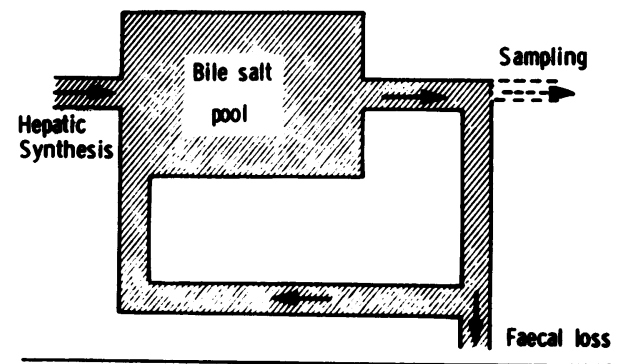

EFFECT OF ACUTE INTERRUPTION OF EHC

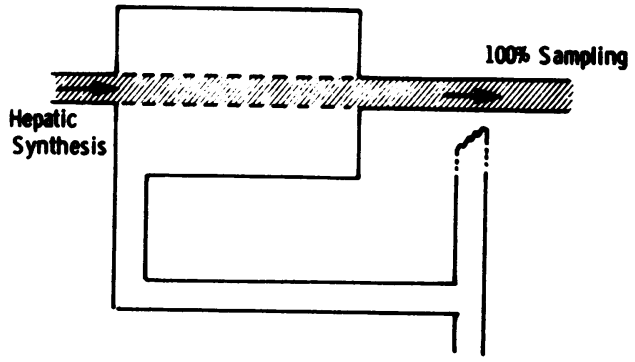

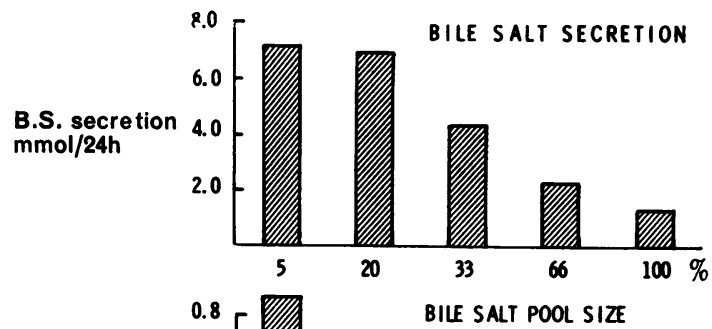

B.S. pool mmol
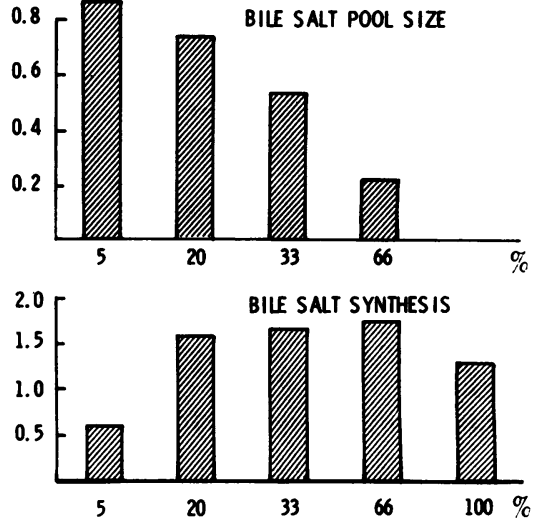

Interruption of EHC

Fig 5

Fig 6

Fig 5 Schematic representation of the principles of measurement of the bile acid pool by the washout technique (Eriksson, 1957). The normal enterohepatic circulation, EHC (upper panel), shows steady state conditions where synthesis matches loss to maintain the normal bile acid pool. In the rhesus monkey model (upper panel), the stream splitter permits sampling of bile (the broken lines).

Following acute interruption of the EHC with $100 \%$ sampling or diversion of bile (lower panel), the bile acid pool is 'washed out' until a point when any bile acids secreted have been newly synthesized by the liver.

Fig 6 Bile acid synthesis rate, pool size, and secretion rate in one animal with varying degrees of interruption of the enterohepatic circulation $(E H C)$.

the enterohepatic circulation may be integrated is illustrated in fig. 6 which shows the bile acid secretion rate, synthesis, and pool size in one animal with varying degrees of interruption of the enterohepatic circulation. As the percentage of bile diverted increased from 5 to 20 , synthesis increased to maintain both pool size and secretion rate essentially unchanged. With greater than $33.3 \%$ biliary diversion, synthesis, although working maximally, was no longer adequate to compensate for bile acid loss, and as a result there was progressive depletion of both bile acid pool size and secretion rate.

\section{Effect of Diet on the Enterohepatic Circulation of Bile Acids}

In studies of the enterohepatic circulation of bile acids in two different centres (Dowling, Mack, and Small, 1970; Campbell et al, 1972a) marked dif- ferences in bile volume and bile acid secretion were found in animals given two different diets (Campbell, Cowley, and Dowling, 1972b). In monkeys given one type of commercial diet (Purina monkey chow) high in fat and calories, both bile volume and bile acid secretion were significantly greater than in animals fed a second commercial diet (diet 41B) with a lower fat and calorie content. When the diets were interchanged between the two groups of animals, the higher fat diet again stimulated both bile volume and bile acid secretion, though there was no change in the size of the bile acid pool.

The effects of supplementing the low-fat diet with individual additives of different lipids were then studied. When the basic low-fat diet was supplemented with the monounsaturated olive oil, so that the total fat intake matched that of the high fat diet, there was no effect on either bile volume or bile acid secretion. However, when the fat supplement was in 


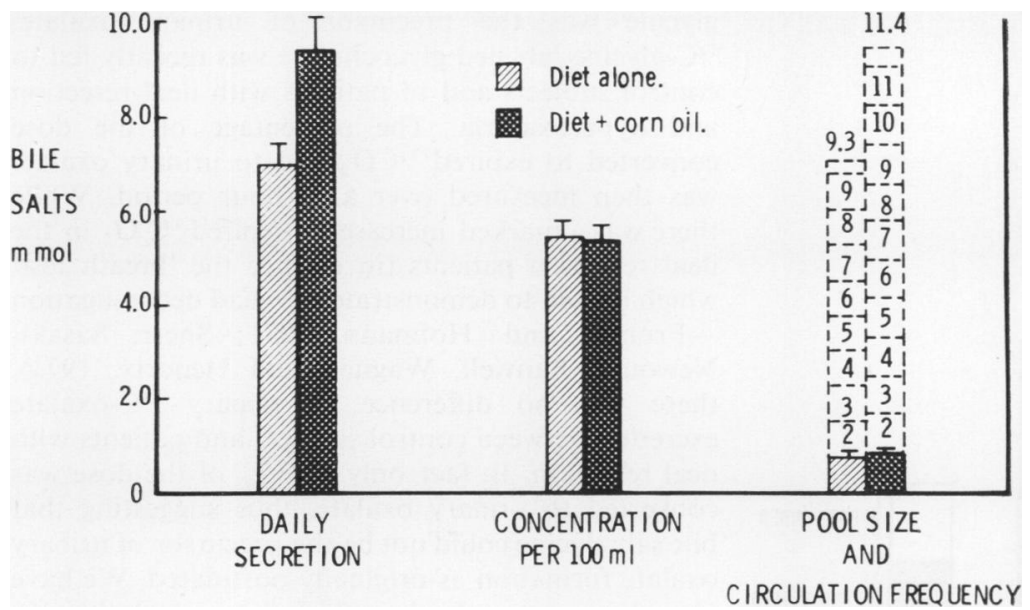

Fig 7 Effect of corn oil supplements on the bile salt secretion rate, concentration, pool size, and circulation frequency (numbered blocks) in rhesus monkeys with an 'intact' enterohepatic circulation. the form of polyunsaturated corn oil, there was an immediate and significant increase, both in bile volume (from 140, SEM \pm 15 , to $170 \pm 16 \mathrm{ml} / 24 \mathrm{hr}$ ) and bile acid secretion $(6.98 \pm 0.46$ increasing to $9.38 \pm 0.76 \mathrm{mmol} / 24 \mathrm{hr}$ ). The greater bile acid secretion rate was not associated with any change in bile acid concentration, but was due to a greater bile volume associated with more frequent circulation of the bile acid pool $(9 \cdot 3$ times $/ 24 \mathrm{hr}$ on the low fat diet and 11.4 times $/ 24 \mathrm{hr}$ when the diet was supplemented with corn oil-fig 7).

Most previous investigators have found that corn oil feeding, either in experimental animals or in man, promotes increased faecal bile acid excretion. Assuming that the intestine absorbs the same proportion of bile acids each circuit, the principal factor governing faecal bile acid excretion is therefore the frequency of circulation of the bile acid pool. The more frequent enterohepatic cycling produced by corn oil feeding, therefore, could well explain the increased faecal bile acid excretion found in previous studies with polyunsaturated fats. In the steady state any increase in faecal bile acid excretion must be matched by a parallel increase in hepatic bile acid synthesis. To test the hypothesis that corn oil feeding can directly stimulate bile acid synthesis, corn oil supplements were fed to monkeys in which bile acid synthesis had previously been maximally induced by $33 \%$ bile diversion. In this situation there was no increase of bile acid secretion rates.

These dietary studies in the Rhesus monkey, therefore, provide indirect evidence that the hypocholesterolaemic action of polyunsaturated fats such as corn oil may be mediated, at least in part, by a more frequent cycling of the bile acid pool with a resultant increase in faecal bile acid loss, a parallel increase in bile acid synthesis and hence in the catabolism of cholesterol.

\section{Effect of Distal Small Bowel Resection on the Bile} Acid Enterohepatic Circulation

The effect of resection of the distal one- or twothirds of the small bowel is illustrated in figure 7 . The removal of the ileum with its active bile acid transport system stimulated bile acid synthesis, but the fall in the percentage of bile acids reabsorbed was such that the net secretion rate was reduced, particularly when a segment of jejunum was resected along with the terminal ileum (two-thirds distal small bowel resection-fig. 1). The reabsorption of small quantities of bile acids after such resection indicates that the ileum is not the exclusive site for bile acid reabsorption, and indeed recent studies in the rat (Perry, White, and Dowling, 1972) have shown first that the jejunum can normally absorb appreciable quantities of bile acids, probably by passive non-ionic diffusion (Small and Dietschy, 1968), and secondly that following ileal resection there is an adaptive increase in jejunal bile acid reabsorption, presumably as a result of 'compensatory' mucosal hyperplasia which increases the absorptive surface.

\section{Clinical Effects of an Interrupted Bile Acid Entero- hepatic Circulation}

SECONDARY HYPEROXALURIA AND RENAL

OXALATE STONE FORMATION IN ILEAL

DISEASE

After ileal resection the circulating bile acids are conjugated almost exclusively with glycine. In the absence of the active transport system in the ileum, bile acid glycine reaching the colon because of ileal malabsorption is liberated by colonic bacteria and converted by further bacterial enzymes to glyoxylate. The latter is then absorbed, transported to the liver, 


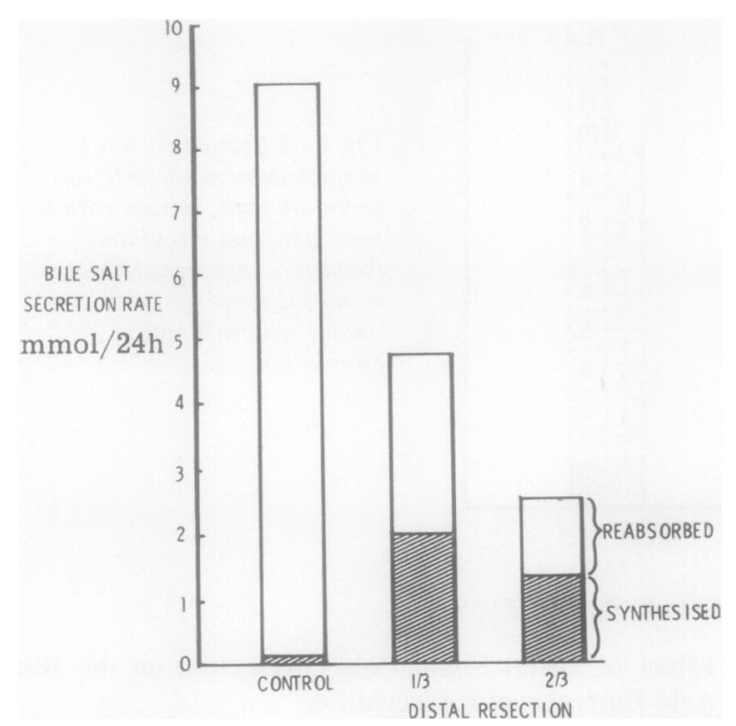

Fig 8 Effect of resection of the distal one or two thirds of small bowel on bile acid secretion rates compared with results in control animals with an 'intact' $(5 \%$ diversion) enterohepatic circulation. The contributions by reabsorption and synthesis are also shown.

oxidized to oxalic acid and excreted by the kidneys to produce hyperoxaluria, and occasionally calcium oxalate renal stones which have recently been observed in several groups of patients with ileal dysfunction (Hofmann, Thomas, Smith, and McCall, 1970; Dowling, Rose, and Sutor, 1971 ; Admirand, Earnest, and Williams, 1971). This, at least, was the original theory proposed to explain the secondary hyperoxaluria which frequently complicates ileal resection.

Our initial experience of this syndrome at the Hammersmith Hospital has recently been expanded, and in a series of 22 patients with ileal disease or resection approximately $66 \%$ of patients had hyperoxaluria as defined by a 24 -hour urinary oxalate excretion exceeding $40 \mathrm{mg} / 24$ hours.

If bile acid glycine was indeed the precursor of urinary oxalate in these patients, then by reducing the proportion of bile acids conjugated with glycine in favour of taurine, one should be able to prevent the increased urinary oxalate excretion. Although taurine feeding has occasionally reduced urinary oxalate excretion (Admirand et al, 1971), we (Chadwick, Modha, and Dowling, 1972a) and others (Smith, Fromm, and Hofmann, 1972) have been unable to confirm our initial observation on one patient (Dowling et al, 1971) that taurine feeding abolishes this secondary form of hyperoxaluria.

To test the original hypothesis that bile acid glycine was the precursor of urinary oxalate, ${ }^{14} \mathrm{C}$-glycine-labelled glycocholate was recently fed to control subjects and to patients with ileal resection and hyperoxaluria. The percentage of the dose converted to expired ${ }^{14} \mathrm{CO}_{2}$ and to urinary oxalate was then measured over a 24-hour period. While there was a marked increase in expired ${ }^{14} \mathrm{CO}_{2}$ in the ileal resection patients (in essence the 'breath test' which is used to demonstrate bile acid deconjugation -Fromm and Hofmann, 1971; Sherr, Sasaki, Newman, Banwell, Wagner, and Hendrix, 1971), there was no difference in urinary ${ }^{14} \mathrm{C}$-oxalate excretion between control subjects and patients with ileal resection. In fact only $0.03 \%$ of the dose was converted to urinary oxalate, thus suggesting that bile salt glycine could not be the precursor of urinary oxalate formation as originally postulated. We have also shown recently that glyoxylate metabolism in these patients is normal. However, when ${ }^{14} \mathrm{C}$-oxalate absorption was measured in control subjects and in ileal resection patients with and without hyperoxaluria, a marked increase in oxalate absorption was found in those with increased urinary oxalate excretion (fig 9). Assuming that dietary oxalate is absorbed to the same extent as the ${ }^{14} \mathrm{C}$-isotope, the enhanced oxalate absorption shown in fig 9 is adequate to explain the hyperoxaluria in patients with this syndrome. A logical conclusion is that such patients should benefit from a low oxalate diet and preliminary results suggest that this is indeed a simple and effective therapy (Chadwick, Modha, and Dowling, 1972b).

Thus, although the original hypothesis that bile acid glycine is the precursor of urinary oxalate in patients with ileal resection does not appear to be correct, altered bile acid metabolism may still explain the hyperoxaluria seen in this syndrome. Physiological concentrations of bile acids in the jejunal lumen have been shown to inhibit water and electrolyte transport, both in experimental animals (Harries and Sladen, 1972; Teem and Phillips, 1972) 윽 and in man (Wingate, Phillips, and Hofmann, 1972). If bile acids exert a similar inhibitory effect on dietary oxalate absorption, then the intraluminal bile acid deficiency which follows ileal resection may $ᄋ$ permit uninhibited oxalate absorption in patients $\tilde{N}$ with ileal resection.

\section{Bile Acids and Gallstones}

In westernized societies the majority of gallstones $\stackrel{\oplus}{+}$ consist predominantly of cholesterol, which is $\frac{T}{T}$ virtually insoluble in water; yet bile, an aqueous $\frac{\vec{O}}{\vec{D}}$ medium, contains a considerable quantity of $\stackrel{\mathbb{Q}}{\stackrel{\leftrightarrow}{\mathscr{Q}}}$ cholesterol which is held in solution by the detergent $\stackrel{\AA}{\AA}$ action of bile acids and phospholipids. Ileal resection 

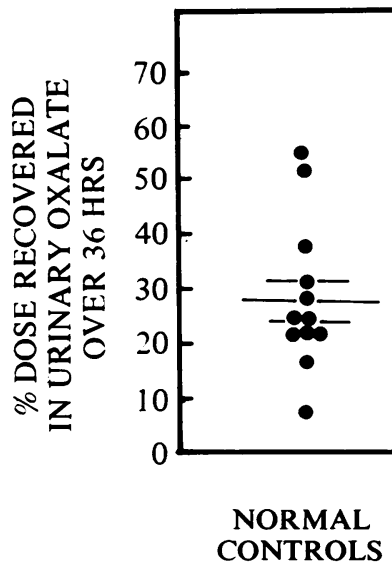

(12)

\section{ILEAL RESECTION ILEAL RESECTION WITH \\ WITHOUT} HYPEROX

(7)

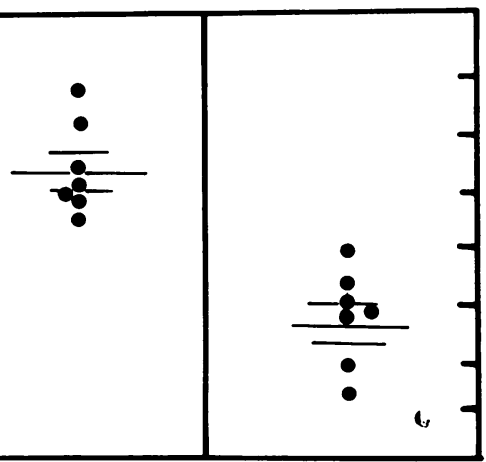

Fig 9 Thirty-six-hour urinary excretion of ${ }^{14} \mathrm{C}$-oxalate in control subjects and in ileal resection patients with and without hyperoxaluria after an oral dose of ${ }^{14} \mathrm{C}$-oxalate (Chadwick et al, 1972a). Horizontal lines show the mean $\pm S E M$.

$$
\begin{array}{ll}
t=5.095 & t=6.075 \\
p<0.001 & p<0.001
\end{array}
$$
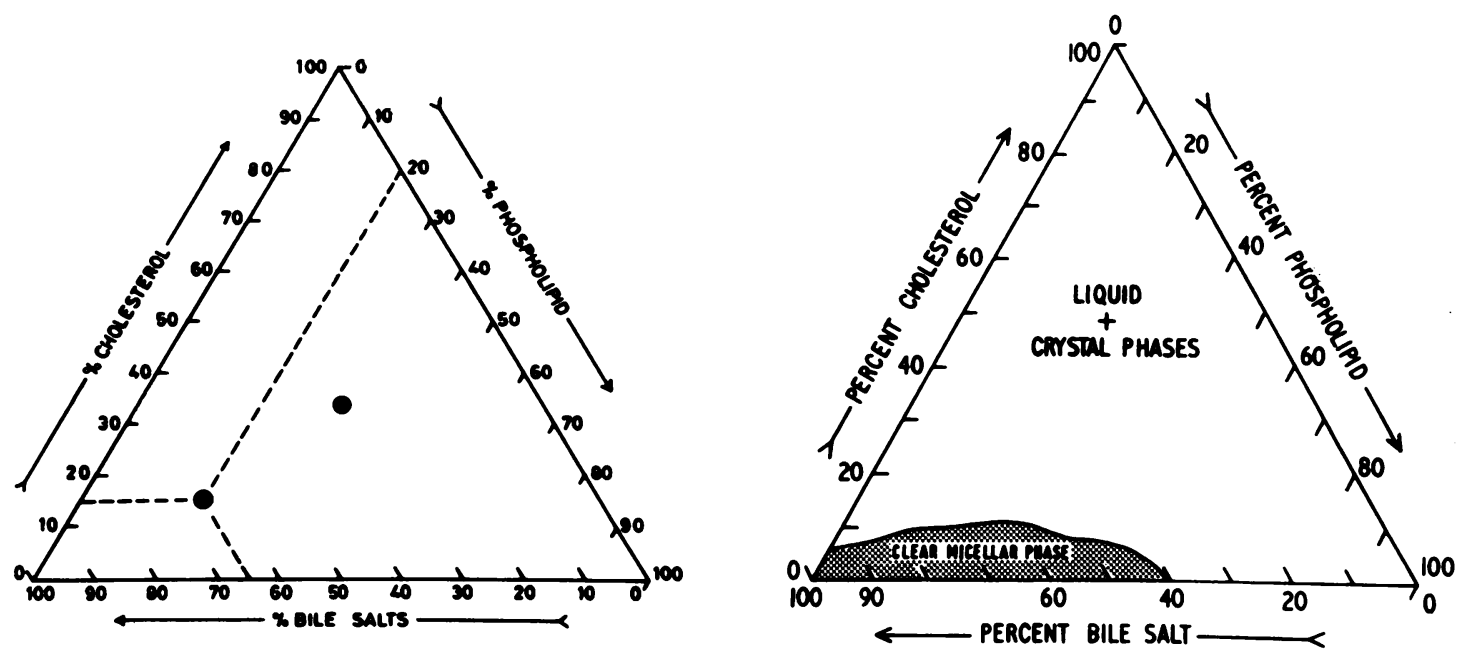

Fig 10a The use of triangular coordinate diagrams to represent the bile salt, phospholipid, and cholesterol content of bile. Any mixture of three components may be represented by a single point on triangular coordinates, provided that the sum of the three components equals 100 , ie, if the concentration of each component (in moles/l) is expressed as a percentage of the total. On the left-hand diagram, a point representing a mixture containing $65 \%$ bile salts, $15 \%$ cholesterol, and $20 \%$ phospholipids is shown. A mixture containing equal amounts of all three variables would be represented by a point falling in the middle of the triangle.

The diagram on the right illustrates cholesterol solubility as determined by Admirand and Small (1968) in artificial mixtures of bile salts, phospholipids, and cholesterol, containing 80-95\% water to simulate gallbladder bile. The small shaded zone in the bottom left of this triangle represents mixtures in which cholesterol was present as a clear, one-phase, micellar solution. Elsewhere in the triangle the proportions of bile salts and phospholipids were insufficient to hold the cholesterol in solution, so that in addition to the supernatant liquid micellar phase, there were now one or more crystalline phases of precipitated cholesterol. The curved boundary of the shaded area, therefore, indicates the limits of cholesterol solubility in bile. Points falling on this line represent a saturated solution. 
causes bile acid malabsorption and, although hepatic bile acid synthesis and jejunal bile acid reabsorption both increase to compensate for bile acid loss, the net effect is depletion of the bile acid pool with reduced bile acid concentrations in bile. The questions arise, therefore, whether ileal disease or resection impair cholesterol solubility in bile and whether patients with Crohn's disease, radiation ileitis, or ileal resection suffer an increased risk of cholesterol gallstone formation.

The composition of bile, as measured in bile-rich duodenal fluid aspirated from the duodenum of fasting subjects following intravenous cholecystokinin, was therefore measured in control subjects and in patients with ileal resection (Dowling, Bell, and White, 1972). In control subjects the bile salt, cholesterol, and phospholipid content of bile when plotted on triangular coordinates as described by Admirand and Small (1968), and illustrated in fig. $10 a$, fell within the limits of cholesterol solubility as

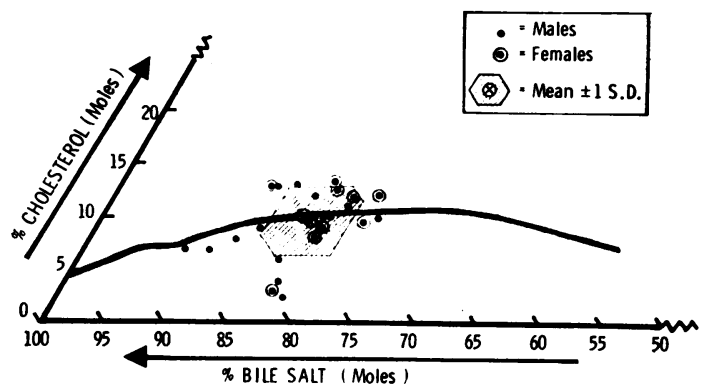

Fig 10b Bile salt, cholesterol, and phospholipid content of bile-rich duodenal fluid in control subjects plotted on triangular coordinates (see fig 10a). Only two axes are shown as the concentration of the third constituent can be derived by subtracting from $100 \%$ the sum of the other two.

The curved line indicates the limits of cholesterol solubility in bile (Admirand and Small, 1968); points falling below the line are normal, while those above the line indicate bile supersaturated with cholesterol.

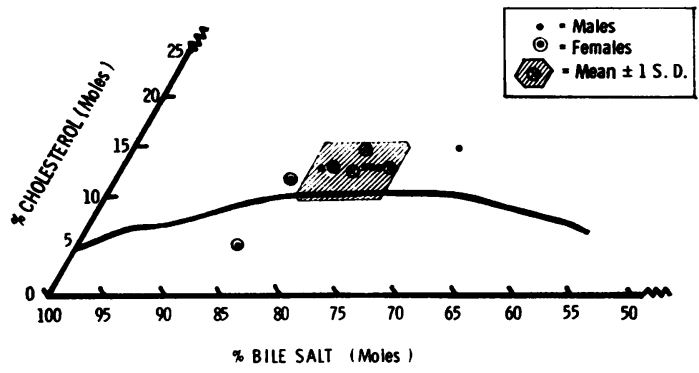

Fig 10c The same as $10 b$ for patients with ileal dysfunction. defined by these authors (fig. 10b). In contrast, in patients with ileal resection all but one had supersaturated bile (fig 10c).

The results of these studies provide a rational explanation for the clinical observation first made by Heaton and Read (1969), and subsequently confirmed by Cohen, Kaplan, Gottlieb, and Patterson (1971), that patients with ileal dysfunction have a much greater prevalence of gallstones than matched control subjects.

The formation of supersaturated bile and the subsequent development of gallstones in patients with ileal dysfunction is an example of secondary cholelithiasis. Such patients have a diminished bile is acid pool. It is probable that diminution of the bile in acid pool size is the basic defect in the formation of $\vec{i}$ supersaturated bile in primary cholelithiasis in 6 patients who have an apparently normal intestinal 옹 tract (Vlahcevic, Bell, Buhac, Farrar, and Swell, 1970; Danzinger, Hofmann, Schoenfield, and Thistle, 1971).

GALLSTONE DISSOLUTION

If depletion of the bile acid pool is the fundamental defect leading to the formation of a supersaturated bile, then expansion of this pool by bile acid feeding should both correct the defect and promote gallstone dissolution. In 1971 Thistle and Schoenfield studied the Chippewa, one of several North American Indian tribes whose women have an extremely high incidence of cholesterol gallstones, and showed that by feeding the primary bile acid, chenodeoxycholic acid, to young Chippewa women with supersaturated bile before they developed gallstones, the abnormality in bile composition could be reversed. In further studies from the Mayo Clinic, Danzinger et al (1972) showed that chenodeoxycholic acid in a dose of $0.75-4.5 \mathrm{~g} /$ day promoted gallstone dissolution in four of seven patients. The results of our own experience with this form of treatment in patients with presumed cholesterol gallstones have recently been published (Bell, Whitney, and Dowling, 1972). Chenodeoxycholic acid, in smaller doses $(0 \cdot 75-1.5 \mathrm{~g} /$ day) than those previously used, enhanced $N$ cholesterol solubility in bile in the majority of patients. Preliminary results after six months of treatment have shown that in a group of 15 patients $\omega$ with gallstones associated with functioning gall- ${ }^{\circ}$ bladders, the stones have disappeared in four patients $\varrho$ with multiple small gallstones. Medium sized stones in four other patients have shown an appreciable reduction in size, while five patients with large, radiolucent stones and two patients with medium to large calcified stones have, as yet, shown no change.

There have been no major complications of this $\stackrel{\mathbb{D}}{\circ}$ form of treatment. However approximately $50 \%$ of 
patients suffer initial diarrhoea, presumably related to exogenous bile acids reaching the colon where they exert a cathartic effect. Hepatic dysfunction is a potential complication of this form of treatment, but apart from a slight increase in the serum isocitrate dehydrogenase (EC 1.1.1.42), which seems of doubtful clinical significance, there have been no other abnormalities of hepatic function tests. Theoretically hypercholesterolaemia might be expected to complicate 'medical' gallstone treatment for two reasons: first, exogenous bile acid feeding might be expected to block endogenous bile acid synthesis from cholesterol; and secondly, bile acid feeding could possibly promote increased dietary cholesterol absorption. However, to date there have been no changes in fasting serum cholesterol levels measured at monthly intervals during treatment.

From the results of these preliminary studies it seems that chenodeoxycholic acid therapy both enhances cholesterol solubility in bile and promotes gallstone dissolution. It remains to be seen whether these beneficial effects are confined to chenodeoxycholic acid, or whether they are shared by other primary and secondary bile acids. The duration of treatment and the optimum dose of bile acid to be used have yet to be established. Whether cessation of therapy will result in a 'relapse' in bile composition and, if so, whether gallstones will re-form are problems which have yet to be answered.

It is a pleasure to acknowledge the stimulation of my former colleague, Dr Donald M. Small from Boston, with whom many of these studies were initiated. I would also like to acknowledge the help and cooperation of my colleagues, Drs Bryan Campbell, Duncan Bell, and Vinton Chadwick, and my technician, Mrs June White, whose work has been quoted in this paper. Mrs Hazel Creed kindly typed the script.

\section{References}

Admirand, W. H., Earnest, D., and Williams, H. E. (1971). Hyperoxaluria and bowel disease. Trans. Ass. Amer. Phycns, 84, 307-312.

Admirand, W. H., and Small, D. M. (1968). The physicochemical basis of cholesterol gallstone formation in man. J. clin. Invest., 47, 1043-1052.

Bell, G. D., Whitney, B., and Dowling, R. H. (1972). Gallstone dissolution in man using chenodeoxycholic acid. Lancet, 2, 1213-1216.

Bouchier, I. A. D., Cooperband, S. R., and Baroukh, M. (1965). Mucous substances and viscosity of normal and pathological human bile. Gastroenterology, 49, 343-353.

Campbell, C. B., Burgess, P., Roberts, S. A., and Dowling R. H. (1972a). The use of rhesus monkeys to study biliary secretion with an intact enterohepatic circulation. Aust. N.Z. J. Med., 2, 49-56.

Campbell, C. B., Cowley, D. J., and Dowling, R. H. (1972b). Dietary factors affecting biliary lipid secretion in the rhesus monkey: a mechanism for the hypocholesterolaemic action of polyunsaturated fat? Europ. J. clin. Invest., 2, 332-341.
Chadwick, V. S., Modha, K., and Dowling, R. H. (1972a). The mechanisms of secondary hyperoxaluria in ileal resection. In Proceedings of the 2nd International Symposium on Bile Acids in Health and Disease, Freiburg, June, 1972. Schattauer, Stuttgart.

Chadwick, V. S., Modha, K., and Dowling, R. H. (1972b). Pathogenesis of secondary hyperoxaluria in ileal resection. (Abstr.) Gut, 13, 840 .

Cohen, S., Kaplan, M., Gottlieb, L., and Patterson, J. (1971). Liver disease and gallstones in regional enteritis. Gastroenterology, 60, 237-245.

Danzinger, R. G., Hofmann, A. F., Schoenfield, L. J., and Thistle, J. L. (1971). Altered bile acid metabolism in patients with cholesterol cholelithiasis. (Abstr.) J. clin. Invest., 50, 24a.

Danzinger, R. G., Hofmann, A. F., Schoenfield, L. J., and Thistle, J. L. (1972). Dissolution of cholesterol gallstones by chenodeoxycholic acid. New Engl. J. Med., 286, 1-8.

Dowling, R. H., Bell, G. D., and White, J. (1972). Lithogenic bile in patients with ileal dysfunction. Gut, 13, 415-420.

Dowling, R. H., Mack, E., Picott, J., Berger, J., and Small, D. M. (1968). Experimental model for the study of the enterohepatic circulation of bile in rhesus monkeys. J. Lab. clin. Med., 72, 169-176.

Dowling, R. H., Mack, E., and Small, D. M. (1970). Effects of controlled interruption of the enterohepatic circulation of bile salts by biliary diversion and by ileal resection on bile salt secretion, synthesis and pool size in the rhesus monkey. $J$. clin. Invest., 49, 232-242.

Dowling, R. H., Rose, G. A., and Sutor, D. J. (1971). Hyperoxaluria and renal calculi in ileal disease. Lancet, 1, 1103-1106.

Eriksson, S. (1957). Biliary excretion of bile acids and cholesterol in bile fistula rats. Bile acids and steroids. Proc. Soc. exp. Biol. $(N . Y), 94,.578-582$.

Fromm, H., and Hofmann, A. F. (1971). Breath test for altered bile acid metabolism. Lancet, 2, 621-625.

Harries, J. T., and Sladen, G. E. (1972). The effects of different bile salts on the absorption of fluid, electrolytes and monosaccharides in the small intestine of the rat in vivo. Gut, 13, 596-603.

Heaton, K. W., and Read, A. E. (1969). Gall-stones in patients with disorders of the terminal ileum and disturbed bile salt metabolism. Brit. med. J., 3, 494-496.

Hofmann, A. F., Thomas, P. J., Smith, L. H., and McCall, J. T. (1970). Pathogenesis of secondary hyperoxaluria in patients with ileal resection and diarrhea. (Abstr.) Gastroenterology, $58,960$.

Lindstedt, S. (1957). The turnover of cholic acid in man: bile acids and steroids 51. Acta physiol. scand., 40, 1-9.

Myant, N. B., and Eder, H. A. (1961). The effect of biliary drainage upon the synthesis of cholesterol in the liver. J. Lipid Res., 2, 363-368.

Perry, P. M., White, J., and Dowling, R. H. (1972). Bile salt absorption following small bowel resection in the rat. (Abstr.) Gut, 13, 845.

Small, N. C., and Dietschy, J. M. (1968). Characterization of the monomer and micelle components of the passive diffusion process of bile acids across the small intestine of the rat. (Abstr.) Gastroenterology, 54, 1272.

Sherr, H. P., Sasaki, Y., Newman, A., Banwell, J. G., Wagner, H. N., Jr., and Hendrix, T. R. (1971). Detection of bacterial deconjugation of bile salts by a convenient breath-analysis technic. New Engl. J. Med., 285, 656-661.

Smith, L. H., Fromm, H., and Hofmann, A. F. (1972). Acquired hyperoxaluria, nephrolithiasis, and intestinal disease. New Engl. J. Med., 286, 1371-1375.

Teem, M. V., and Phillips, S. F. (1972). Perfusion of the hamster jejunum with conjugated and unconjugated bile acids: inhibition of water absorption and effects on morphology. Gastroenterology, 62, 261-267.

Thistle, J. L., and Schoenfield, L. J. (1971). Induced alterations in composition of bile of persons having cholelithiasis. Gastroenterology, 61, 488-496.

Vlahcevic, Z. R., Bell, C. C., Jr., Buhac, I., Farrar, J. T., and Swell, L. (1970). Diminished bile acid pool size in patients with gallstones. Gastroenterology, 59, 165-173.

Wingate, D. L., Phillips, S. F., and Hofmann, A. F. (1972). The effect of conjugated bile acids and lecithin on water and glucose absorption in the human jejunum. (Abstr.) Biol. et Gastroent., $5,633 \mathrm{C}$. 